Joint analysis of longitudinal feed intake and single recorded production traits in pigs using a novel horizontal model

Shirali, M.; Strathe, A. B.; Mark, T.; Nielsen, B.; Jensen, J.

Published in:

Journal of Animal Science

DOI:

10.2527/jas2016.0606

Publication date:

2017

Document version

Publisher's PDF, also known as Version of record

Document license:

Unspecified

Citation for published version (APA):

Shirali, M., Strathe, A. B., Mark, T., Nielsen, B., \& Jensen, J. (2017). Joint analysis of longitudinal feed intake and single recorded production traits in pigs using a novel horizontal model. Journal of Animal Science, 95(3), 1050-1062. https://doi.org/10.2527/jas2016.0606 


\title{
Joint analysis of longitudinal feed intake and single recorded production traits in pigs using a novel Horizontal model ${ }^{1}$
}

\author{
M. Shirali, ${ }^{2,3}$ A. B. Strathe, $\uparrow \dagger^{2}$ T. Mark, $\dagger$ B. Nielsen, $\uparrow$ and J. Jensen* \\ *Department of Molecular Biology and Genetics, Aarhus University, Blichers Allé 20, Tjele, \\ Denmark, 8830; †Section of Animal Genetics, Bioinformatics and Breeding, Department of Veterinary Clinical \\ and Animal Sciences, Faculty of Health and Medical Sciences, University of Copenhagen, Frederiksberg, Denmark, \\ 1870 Frederiksberg C.; and $\$$ Pig Research Centre, Danish Agriculture and Food Council, Copenhagen, Denmark, 1620
}

\begin{abstract}
A novel Horizontal model is presented for multitrait analysis of longitudinal traits through random regression analysis combined with single recorded traits. Weekly ADFI on test for Danish Duroc, Landrace, and Yorkshire boars were available from the national test station and were collected from 30 to $100 \mathrm{~kg} \mathrm{BW}$. Single recorded production traits of ADG from birth to $30 \mathrm{~kg} \mathrm{BW}$ (ADG30), ADG from 30 to $100 \mathrm{~kg}$ BW (ADG100), and lean meat percentage (LMP) were available from breeding herds or the national test station. The Horizontal model combined random regression analysis of feed intake (FI) with single recorded traits of ADG100, LMP, and ADG30. In the Horizontal model, the FI data were horizontally structured with FI on each week as a "trait." The additive genetic and litter effects were modeled to be common across different FI records by reducing the rank of the covariance matrices using second- and first-order Legendre polynomials of age on test, respectively. The fixed effect and random residual variance were estimated for each weekly FI trait. Residual feed intake (RFI) was derived from the conditional distribution of FI given the breeding values of ADG100 and LMP. The heritability of FI varied by week on test in Duroc (0.12 to 0.19$)$, Landrace (0.13 to 0.22$)$, and Yorkshire

( 0.21 to 0.23$)$. The heritability of RFI was lowest and highest in wk $6(0.03)$ and $10(0.10)$, respectively, in Duroc and wk 7 (0.04 and 0.02) and 1 (0.09 and 0.20), respectively, in Landrace and Yorkshire. The proportion of FI genetic variance explained by RFI ranged from 20 to $75 \%$ in Duroc, from 19 to $75 \%$ in Landrace, and from 11 to $91 \%$ in Yorkshire. Average daily gain from 30 to $100 \mathrm{~kg} \mathrm{BW}$ and ADG30 heritabilities were moderate in Duroc (0.24 and 0.22, respectively), Landrace ( 0.34 and 0.25 , respectively), and Yorkshire (0.34 and 0.22 , respectively). Lean meat percentage heritability was moderate in Duroc $(0.37)$ and large in Landrace (0.62) and Yorkshire (0.60). The genetic correlation of FI with ADG100 increased by week on test followed by a $32 \%$ decrease from wk 7 in Duroc and a $7 \%$ decrease in dam line breeds. Defining RFI as genetically independent of production traits leads to consistent and easy interpretable breeding values. The genetic parameters of traits in the feed efficiency complex and their dynamics over the test period showed breed differences that could be related to the fatness and growth potential of the breeds. The Horizontal model can be used to simultaneously analyze repeated and single recorded traits through proper modeling of the environmental variances and covariances.
\end{abstract}

Key words: feed efficiency, multitrait analysis, pigs, random regression, variance components

\footnotetext{
${ }^{1}$ Authors acknowledge the European Union Seventh Framework Programme (FP7/2007-2013) for funding ECO-FCE project (grant agreement number 311794) under which this research took place. Authors are also grateful for funding from Danish Strategic Research Council (GenSAP; contract number 12-132452) and Danish Ministry of Food, Agriculture and Fisheries (GUDP; grant number 34009-14-
}

0849). Authors disclosing no potential or actual conflict of interested related to the research presented in the manuscript.

${ }^{2}$ These authors share the first authorship.

${ }^{3}$ Corresponding author: Mahmoud.Shirali@mbg.au.dk

Received May 5, 2016.

Accepted December 30, 2016. 


\section{INTRODUCTION}

Random regression models are common for analyzing longitudinal data in livestock (Schaeffer, 2004). Joint analysis of longitudinal and single recorded traits requires a multitrait setup to properly account for environmental covariance. This is because environmental covariance is modeled into permanent and measurement error in random regression models but only residual dispersion in single recorded trait models. Schnyder et al. (2002) proposed a multitrait model for such joint analysis by introducing a permanent environment effect and forcing the residual variance to be very small. Such a model has unrealistic assumptions; for example, all environmental variance is permanent and all environmental covariances are between the residual of single recorded traits and permanent effect of longitudinal trait. This could lead to biased estimation of genetic parameters and breeding values (Schnyder et al., 2002). Here, we present a novel model (termed Horizontal model) to ensure proper modeling of genetic and environmental covariances between longitudinal and single recorded traits. Under the Horizontal model, the vector of residuals for the longitudinal trait is modeled as the sum of permanent effects due to the animal and temporary residual, avoiding partitioning of variance due to permanent and temporary environmental effects. In animal breeding, feed intake data is collected longitudinally along with single recorded performance traits. Residual feed intake as partial measure of feed efficiency has shown to have a varying genetic background during growth in pigs (Strathe et al., 2014). The aims of the study were to 1) present a novel approach (Horizontal model) for combined analysis of longitudinal and single recorded traits, 2) investigate the dynamics of genetic parameters for feed intake and residual feed intake during the growth of pigs in 3 Danish pig breeds, and 3) derive longitudinal genetic residual feed intake from the longitudinal model for feed intake.

\section{MATERIAL AND METHODS}

Animal Care and Use Committee approval was not obtained for this study because the data were obtained from an existing database of performance records.

\section{Data}

Data for this study were supplied by Pig Research Centre, Danish Agriculture and Food Council (Brussels, Denmark), and were part of the Danish pig breeding program routinely recording data in nucleus breeding herds and at the national test station (Kjellerup, Denmark.). Feed intake (FI) records of 10,911, 6,691, and 6,632
Danish breeds of Duroc, Landrace, and Yorkshire boars, respectively, were recorded at the national Danish test station (Bøgildgård; Kjellerup, Denmark) from approximately 30 to $100 \mathrm{~kg} \mathrm{BW}$, using ACEMA64 electronic feeders (ACEMO, Pontivy, France) that recorded FI at each visit to the feeding station. Feed intake data were available in the period from October 2008 to December 2013. The piglets (approximately $7 \mathrm{~kg}$ ) came from different nucleus breeding herds and were reared under controlled climate conditions to $25 \mathrm{~kg}$; then, pigs were moved to performance test barns and given approximately 1 wk of adaptation to the automatic feed dispensers before the performance test period commenced. The records from the adaptation period were discarded. Pigs were fed ad libitum during the test period. Raw data contained FI records per visit to the feeding machine during the performance test period. Then, daily FI was obtained as sum of FI records on a daily basis. Week on test was defined as each 7-d period on the test from the date an animal completed the adaptation period. Furthermore, ADFI in each week during the performance test period was calculated and saved as the record for analysis; this was in order to reduce the amount of data for the statistical analyses while also reducing noise in the data. The last FI of an animal were for the week before the end of the performances test, which is the time that the average pig within a pen reaches 100 $\mathrm{kg} \mathrm{BW}$. This resulted in a maximum of 10 FI records for pigs during the total performance test period.

Production traits of ADG from 30 to $100 \mathrm{~kg} \mathrm{BW}$ (ADG100; g/d) as well as lean meat percentage (LMP; \%) were available from 67,638, 121,157, and 145,941 Duroc, Landrace, and Yorkshire performance-tested pigs, respectively, from both the nucleus herds and the national test station. The production trait of ADG from birth to $30 \mathrm{~kg} \mathrm{BW}$ (ADG30; g/d) was recorded only in the nucleus herds; therefore, 54,119 records in Duroc, 113,159 in Landrace, and 138,017 in Yorkshire were available for this trait. The LMP was predicted at $100 \mathrm{~kg}$ BW based on ultrasound records of back fat thickness and BW at the time of ended performance test, using a linear prediction equation that was estimated from previous trials where the ratio of total meat in dissected carcasses was obtained. The data from the breeding herds consisted of records on both intact males and females. Data were edited such that all nucleus breeding herds have recordings during the entire period and the contemporary group (year $\times$ herd $\times$ month on test) sizes consisted of at least 20 records.

Descriptive statistics for FI and the production traits included in the data set are presented in Table 1. Most FI records were available for the terminal breed, Duroc, whereas the Landrace and Yorkshire breeds had more production trait records. On average, Duroc 
Table 1. Descriptive statistics for feed intake (FI) from wk 1 to 10 on test (FI. \{week\}) and ADG from birth to 30 $\mathrm{kg} \mathrm{BW}$ (ADG30) and from 30 to $100 \mathrm{~kg} \mathrm{BW} \mathrm{(ADG100)} \mathrm{as} \mathrm{well} \mathrm{as} \mathrm{lean} \mathrm{meat} \mathrm{percentage} \mathrm{(LMP)} \mathrm{in} \mathrm{Danish} \mathrm{Duroc,}$ Landrace, and Yorkshire breeds

\begin{tabular}{|c|c|c|c|c|c|c|c|c|c|c|c|c|c|c|c|c|c|c|}
\hline \multirow[b]{3}{*}{ Trait } & \multicolumn{18}{|c|}{ Breed $^{1}$} \\
\hline & \multicolumn{6}{|c|}{ Duroc } & \multicolumn{6}{|c|}{ Landrace } & \multicolumn{6}{|c|}{ Yorkshire } \\
\hline & No. & Mean & $\mathrm{CV}$ & SD & Min. & Max. & No. & Mean & $\mathrm{CV}$ & $\mathrm{SD}$ & Min. & Max. & No. & Mean & $\mathrm{CV}$ & SD & Min. & Max. \\
\hline$\overline{\text { FI.1 }}$ & 10,913 & 1,358 & 0.25 & 341 & 292 & 2,428 & 6,686 & 1,350 & 0.28 & 381 & 178 & 2,539 & 6,632 & 1,336 & 0.17 & 233 & 594 & 2,069 \\
\hline FI.2 & 10,890 & 1,646 & 0.21 & 338 & 558 & 2,716 & 6,673 & 1,648 & 0.22 & 355 & 524 & 2,758 & 6,635 & 1,544 & 0.16 & 241 & 774 & 2,296 \\
\hline FI.3 & 10,913 & 1,860 & 0.19 & 348 & 753 & 2,952 & 6,675 & 1,857 & 0.18 & 341 & 774 & 2,906 & 6,642 & 1,732 & 0.15 & 256 & 918 & 2,524 \\
\hline FI. 4 & 10,934 & 2,122 & 0.18 & 373 & 941 & 3,270 & 6,681 & 2,063 & 0.17 & 355 & 928 & 3,172 & 6,635 & 1,909 & 0.15 & 279 & 1,017 & 2,787 \\
\hline FI.5 & 10,921 & 2,392 & 0.16 & 390 & 1,122 & 3,587 & 6,675 & 2,276 & 0.16 & 366 & 1,109 & 3,408 & 6,634 & 2,071 & 0.15 & 311 & 1,063 & 3,043 \\
\hline FI.6 & 10,918 & 2,636 & 0.16 & 411 & 1,300 & 3,906 & 6,671 & 2,478 & 0.15 & 379 & 1,237 & 3,682 & 6,620 & 2,234 & 0.16 & 351 & 1,080 & 3,350 \\
\hline FI.7 & 10,840 & 2,879 & 0.15 & 446 & 1,392 & 4,291 & 6,673 & 2,662 & 0.15 & 399 & 1,373 & 3,878 & 6,630 & 2,411 & 0.16 & 393 & 1,127 & 3,649 \\
\hline FI. 8 & 10,135 & 3,102 & 0.16 & 491 & 1,450 & 4,673 & 6,490 & 2,868 & 0.15 & 425 & 1,462 & 4,232 & 6,569 & 2,634 & 0.17 & 439 & 1,179 & 4,027 \\
\hline FI.9 & 7,213 & 3,319 & 0.17 & 554 & 1,354 & 5,161 & 5,716 & 3,096 & 0.15 & 461 & 1,501 & 4,610 & 6,227 & 2,852 & 0.17 & 481 & 1,249 & 4,337 \\
\hline FI.10 & 2,116 & 3,473 & 0.19 & 652 & 1,405 & 5,489 & 3,392 & 3,243 & 0.17 & 538 & 1,303 & 5,001 & 5,070 & 3,023 & 0.18 & 535 & 1,226 & 4,707 \\
\hline ADG1 & 67,638 & 1,081 & 0.10 & 109 & 717 & 1,543 & 121,157 & 999 & 0.12 & 115 & 553 & 1,445 & 145,941 & 950 & 0.11 & 105 & 506 & 1,335 \\
\hline LMP & 67,638 & 61.2 & 0.02 & 0.96 & 56.9 & 64.9 & 121,157 & 62.3 & 0.02 & 1.15 & 56.5 & 66.1 & 145,941 & 61.7 & 0.01 & 0.73 & 57.5 & 64.3 \\
\hline ADG30 & 54,119 & 399 & 0.11 & 42.0 & 286 & 600 & 113,159 & 384 & 0.10 & 37.0 & 295 & 559 & 138,017 & 363 & 0.10 & 35.3 & 283 & 548 \\
\hline
\end{tabular}

${ }^{1}$ No. $=$ number of records; Min. $=$ minimum; Max. $=$ maximum

pigs had higher growth rate and FI than Landrace and Yorkshire breeds. The pedigree for the animals with data was traced back to January 1, 1984, and included 81,200, 130,938, and 155,301 pigs for Duroc, Landrace, and Yorkshire, respectively.

\section{The Horizontal Multitrait Model}

In the Horizontal model, all records on an animal are treated simultaneously, compared with longitudinal random regression models, where the data are usually treated per time point. The following Horizontal multivariate mixed linear model was used to analyze the data:

$$
\begin{aligned}
& \mathbf{y}_{w=1}^{10}=\mathbf{x}_{w=1}^{10} \mathbf{b}_{w=1}^{10}+\mathbf{Z}_{\mathbf{a}^{10}=1} \mathbf{a}_{\mathrm{H}}+\mathbf{Z}_{\mathbf{c}^{10}=1} \mathbf{c}_{\mathrm{H}}+\mathbf{e}_{w=1}^{10}, \\
& \mathbf{y}_{11}=\mathbf{X}_{11} \mathbf{b}_{11}+\mathbf{Z}_{\mathbf{a}_{11}} \mathbf{a}_{11}+\mathbf{Z}_{\mathbf{c}_{11}} \mathbf{c}_{11}+\mathbf{Z}_{\mathbf{p}_{11}} \mathbf{p}_{11}+\mathbf{e}_{11}, \\
& \mathbf{y}_{12}=\mathbf{X}_{12} \mathbf{b}_{12}+\mathbf{Z}_{\mathbf{a}_{12}} \mathbf{a}_{12}+\mathbf{Z}_{\mathbf{c}_{12}} \mathbf{c}_{12}+\mathbf{Z}_{\mathbf{p}_{12}} \mathbf{p}_{12}+\mathbf{e}_{12}, \quad \text { and } \\
& \mathbf{y}_{13}=\mathbf{X}_{13} \mathbf{b}_{13}+\mathbf{Z}_{\mathbf{a}_{13}} \mathbf{a}_{13}+\mathbf{Z}_{\mathbf{c}_{13}} \mathbf{c}_{13}+\mathbf{e}_{13},
\end{aligned}
$$

in which $\mathbf{y}_{w=1}^{10}$ is the multiple FI records for all animals with 1 record for each week on performance test $(\mathrm{w}=1$ to 10 for each week of feed recorded per animal, specifying 10 submodels) and $\mathbf{b}_{w=1}^{10}$ contains effects of year and week on test interactions along with starting BW for each week's FI records. $\mathbf{a}_{\mathrm{H}}$ contains $n r+1$ additive genetic regression coefficients for each animal obtained by modeling common genetic effects across the
FI models ( $\mathrm{w}=1$ to 10$), \mathbf{c}_{\mathrm{H}}$ contains $\mathrm{nf}+1$ regression coefficients of litter effect for each animal obtained by modeling common litter effects across the FI models, and $n r$ and $n f$ are the order of Legendre polynomials (second and first, respectively) fitted as random regression models for animal and litter effects; that is, 3 and 2 parameters are modeled for each animal for additive genetic and litter effects, respectively. Thus, $\mathbf{a}_{\mathrm{H}}=\left[\mathbf{a}_{\mathrm{H}}\right.$, $\left.i, \mathbf{a}_{\mathrm{H}, l}, \mathbf{a}_{\mathrm{H}, q}\right]$ and $\mathbf{c}_{\mathrm{H}}=\left[\mathbf{c}_{\mathrm{H}, i}, \mathbf{c}_{\mathrm{H}, l}\right]$, in which $i, l$, and $q$ indicate intercept, linear, and quadratic terms, respectively. The Legendre polynomials were evaluated at standardized ages. The vector of residuals $\mathbf{e}_{w=1}^{10}$ contains the environmental effects on each week on test. The vectors $\mathbf{y}_{11}, \mathbf{y}_{12}$, and $\mathbf{y}_{13}$ are the phenotypic records of ADG100, LMP, and ADG30, respectively; $\mathbf{b}_{11}$ and $\mathbf{b}_{12}$ contain effects of interactions between year, herd, and month on test in addition to starting BW and gender; $\mathbf{b}_{13}$ contains effects of interactions between year, herd, and month of birth along with initial BW, gender, age of the dam, and parity of the dam of animal; $\mathbf{a}_{11}, \mathbf{a}_{12}$, and $\mathbf{a}_{13}$ contain the additive genetic effect for each animal; $\mathbf{c}_{11}, \mathbf{c}_{12}$, and $\mathbf{c}_{13}$ contain effect of litter; $\mathbf{p}_{11}$ and $\mathbf{p}_{12}$ contain the pen effects for each animal; and $\mathbf{e}_{11}, \mathbf{e}_{12}$, and $\mathbf{e}_{13}$ are the random residuals for each animal. Inclusion of each random effect in the model for each trait was determined by a log likelihood ratio test comparing models with or without each effect included in the model specification; for example, pen effect was not significant for FI measurements. The order of Legendre polynomials were decided based on a log likelihood ratio test comparing models where $n r$ and $n f$ were varied. This model formulation merges random factors across longitudinal FI records. These 
records are treated simultaneously; hence, we termed this model a Horizontal model for short.

The additive genetic animal effect for $\mathbf{y}_{w=1}^{10}$ was modeled across FI traits (weeks on test) using Legendre polynomials of order $n r=2$, which includes an intercept, a linear, and a quadratic term for each animal, whereas the additive genetic effects for $\mathbf{y}_{11}, \mathbf{y}_{12}$, and $\mathbf{y}_{13}$ included only a single breeding value (intercept). The distribution of additive genetic effects were assumed to be

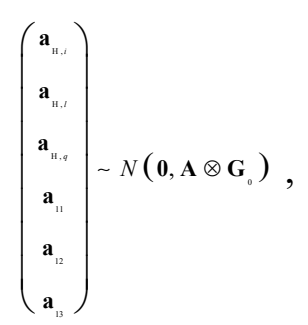

in which $\mathbf{A}$ was the numerator relationship matrix and $\mathbf{G}_{0}$ was a $6 \times 6$ matrix of additive genetic variances and covariances to be estimated,

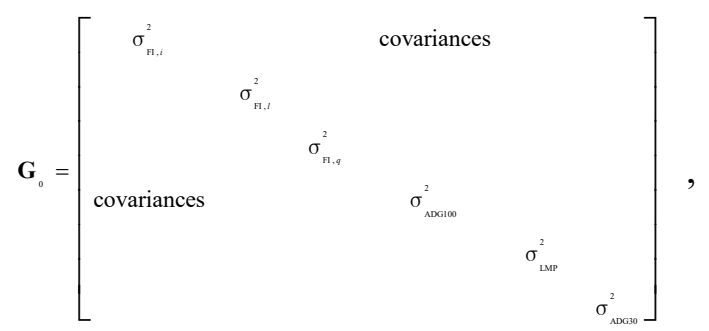

with 21 genetic variance and covariance parameters to be estimated. The first 3 additive genetic variances in $\mathbf{G}_{0}$ are due to intercept $\left(\sigma_{\mathrm{F}, i}^{2}\right)$, linear $\left(\sigma_{\mathrm{FI}, l}^{2}\right)$, and quadratic $\left(\sigma_{\mathrm{FI}, q}^{2}\right)$ effects in the Legendre polynomials and the remaining 3 are the additive genetic variance for ADG100, LMP, and ADG30, respectively.

The distribution of the litter effect was

$$
\left(\begin{array}{c}
\mathbf{c}_{\mathrm{w}, t} \\
\mathbf{c}_{\mathrm{u}, t} \\
\mathbf{c}_{\mathrm{n}} \\
\mathbf{c}_{\mathrm{k}} \\
\mathbf{c}_{\mathrm{k}}
\end{array}\right) \sim N\left(\mathbf{0}, \mathbf{I} \otimes \mathbf{C}_{\mathrm{o}}\right)
$$

in which the structure of $\mathbf{C}_{0}$ was similar to that of $\mathbf{G}_{0}$, with exception that only intercept and linear terms of Legendre polynomials were used for modeling the litter effect. The pen of rearing had an effect only on ADG100 $\left(\mathbf{y}_{11}\right)$ and LMP $\left(\mathbf{y}_{12}\right)$ with distribution assumption of $\left(\begin{array}{l}\mathbf{p}_{11} \\ \mathbf{p}_{12}\end{array}\right) \sim N\left(\mathbf{0}, \mathbf{I} \otimes \mathbf{P}_{0}\right)$, in which $\mathbf{P}_{0}$ is a $2 \times 2$ variance and covariance matrix of pen effects on ADG100 and LMP traits.

Due to the repeated records in FI, the residual variance and covariance $(\mathbf{R})$ had a special structure:

$$
\left(\begin{array}{c}
\mathbf{e}^{10} \\
\mathbf{e}^{10-1} \\
\mathbf{e}_{12} \\
\mathbf{e}_{13}
\end{array}\right) \sim N(\mathbf{0}, \mathbf{R}),
$$

in which $\mathbf{R}$ is thus a $13 \times 13$ matrix:

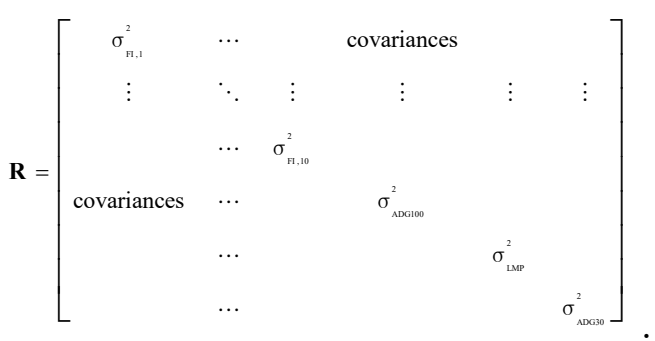

The residual covariances between FI traits and ADG30 were not estimable, as the animals with FI records did not have ADG30 records. Compared with, for example, Schnyder et al. (2002), the advantage of this parametrization is that there is no need to explicitly model repeated measures of the same animal. Instead, all environmental variances and covariances can be correctly specified. Such a covariance structure is also known as compound symmetry (Littell et al., 2000) and can also be specified for other random parts of the model.

In practice, this model representation was achieved by treating all FI measurements for an animal simultaneously allowing for common effects across several measurements in the data, hence the term Horizontal model. The fixed and random effects can then be modeled to be common across traits; that is, the additive genetic effects were modeled to be common across different FI records by forcing the covariance matrices to have reduced rank describing the FI curve by intercept, linear, and quadratic functions. Therefore, the repeated records are modeled horizontally (within animal) instead of longitudinally (across records). If only repeated records are in the model, there is no difference between Horizontal and standard longitudinal models. The module DMUAI within the DMU software package (Madsen and Jensen, 2014) was used to estimate variance and covariance components of the above Horizontal model, and the modeling of common random effects in the model or modelsfor FI was achieved using the "REDUCE" option in the software.

\section{Covariance Function and Derivation of Genetic Residual Feed Intake}

The genetic covariance functions for FI, ADG100, LMP, and ADG30 were derived from the true parameters and then used in the estimated parameters as follows:

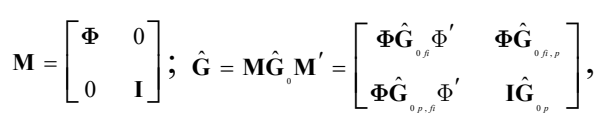


in which $\mathbf{M}$ is a matrix containing the Legendre polynomials coefficients $(\boldsymbol{\Phi})$ for FI and an identity matrix (I) for the single recorded traits. The matrix $\boldsymbol{\Phi}$ of the Legendre polynomials was chosen to be of a second order evaluated at standardized ages $(80,87,94,101,108,115$, $122,129,134$, and $137 \mathrm{~d}$ of age) for Duroc and $(84,91$, $98,105,112,119,126,133,139$, and $143 \mathrm{~d}$ of age) for Landrace and Yorkshire, representing the midpoint age of all animals in the respective weeks on test. Midpoint ages were the time points with larger frequency of recorded data; therefore, they allow for more accurate parameter estimation and avoid any border effects or overparameterization. However, the late weeks on test are not equidistance, which should be taken into account in result interpretation. Therefore, if $\hat{\mathbf{G}}$ is split into its submatrices corresponding to variances and covariances of regression coefficients for FI $\left(\hat{\mathbf{G}}_{0 f i}\right)$, variances and covariances of single measured traits $\left(\hat{\mathbf{G}}_{0 p}\right)$ and variances and covariances between regression coefficients and single measured traits $\left(\hat{\mathbf{G}}_{0 f i, p}\right)$, then $\hat{\mathbf{G}}$ represents all genetic variances and covariances between FI evaluated at desired weeks on test and ADG100, LMP, and ADG30.

The genetic and phenotypic variances of residual feed intake (RFI) as a measure of partial feed efficiency were defined as the covariances of FI in each week on test, conditional on the additive genetic effect of ADG100 and LMP. This is usually termed genetic RFI (Kennedy et al., 1993) using the following covariance function:

$$
\begin{aligned}
& {\left[\begin{array}{cc}
\hat{\mathbf{G}}_{R F I} & \hat{\mathbf{G}}_{R F I, p} \\
\hat{\mathbf{G}}_{p, R F I} & \hat{\mathbf{G}}_{p}
\end{array}\right]=\mathbf{B} \hat{\mathbf{G}} \mathbf{B}^{\prime} \text { and }} \\
& {\left[\begin{array}{cc}
\hat{\mathbf{P}}_{R F I} & \hat{\mathbf{P}}_{R F I, p} \\
\hat{\mathbf{P}}_{p, R F I} & \hat{\mathbf{P}}_{p}
\end{array}\right]=\mathbf{B} \hat{\mathbf{P}} \mathbf{B}^{\prime},}
\end{aligned}
$$

in which $\hat{\mathbf{G}}$ and $\hat{\mathbf{P}}$ are $13 \times 13$ genetic and phenotypic variance and covariance matrices for FI in $10 \mathrm{wk}$ and production traits of ADG100, LMP, and ADG30, respectively.

Genetic RFI was obtained using $\mathbf{B}=\left[\begin{array}{ccc}\mathbf{I}_{F I} & -\hat{\mathbf{b}}_{f \mid p 1} & 0 \\ 0 & \mathbf{I}_{p 1} & 0 \\ 0 & 0 & \mathbf{I}_{p 2}\end{array}\right]$,

with $\hat{\mathbf{b}}_{f i p 1}$ being the multivariate partial genetic regression coefficients for FI on 2 production traits ofADG100 and LMP for each week on the test. Partial genetic regression coefficients were computed based on the estimated variance and covariance components as follows: $\hat{\mathbf{b}}_{f i p 1}=\hat{\mathbf{G}}_{f i, p 1} \hat{\mathbf{G}}_{p 1}^{-1}$, in which $\hat{\mathbf{G}}_{p 1}$ is the genetic variances and covariances for ADG100 and LMP and $\hat{\mathbf{G}}_{f, p 1}$ is the covariances between FI and production traits of ADG100 and LMP from the $\hat{\mathbf{G}}$ matrix. The identity matrix $\mathbf{I}_{F I}$ is of order 10, corresponding to the number of weeks on test, $\mathbf{I}_{p 1}$ is an identity matrix of order 2 for production traits of ADG100 and LMP used for estimation of RFI with the size of $2 \times 2$, and
$\mathbf{I}_{p 2}$ is an identity matrix of order 1 for production traits of ADG30. The matrices $\hat{\mathbf{G}}_{R F I}$ and $\hat{\mathbf{P}}_{R F I}$ are the genetic and phenotypic variance and covariance for RFI estimated at each week on test, respectively, and $\hat{\mathbf{G}}_{R F I, p}$ or $\hat{\mathbf{G}}_{p, R F I}$ and $\hat{\mathbf{P}}_{R F I, p}$ or $\hat{\mathbf{P}}_{p, R F I}$ are the genetic and phenotypic covariances of RFI at different weeks on test. The production traits of ADG100 and LMP have zero genetic correlations with $\operatorname{RFI}\left(\hat{\mathbf{G}}_{R F I, p 1}=\hat{\mathbf{G}}_{f i, p 1}-\hat{\mathbf{b}}_{f i \mid p 1} \hat{\mathbf{G}}_{p 1}=0\right)$, as RFI is defined by partial genetic coefficients for FI on production traits.

Covariance functions were used to obtain population parameters for total FI and RFI during the first to fifth week on test by using $\sum_{w=1}^{5} \phi_{w j}$ for FI during wk 1 to 5 (FI.P1) and RFI during wk 1 to 5 (RFI.P1), during the 6th to 10 th week on test by using $\sum_{w=6}^{10} \phi_{w j}$ for FI during wk 6 to 10 (FI.P2) and RFI during wk 6 to 10 (RFI.P2), and FI during wk 1 to 10 (FI.TP) and RFI during wk 1 to 10 (RFI.TP) by using $\sum_{w=1}^{10} \phi_{w j}$, in which $\phi_{w j}$ is a function of Legendre polynomials evaluated at week $w$ and with order $j$, in which $j=\{0,1,2\}$. Furthermore, the predicted breeding value for genetic RFI for the $i$ th animal at each week on test $(w)$ can be obtained as $\hat{a}_{i w}^{\mathrm{RFI}}=\hat{a}_{i w}^{\mathrm{FI}}-\hat{b}_{1 w} \hat{a}_{i}^{\mathrm{ADG} 100}-\hat{b}_{2 w} \hat{a}_{i}^{\mathrm{LMP}}$, in which $\hat{b}_{1 w}$ and $\hat{b}_{2 w}$ are the partial genetic regression coefficients for ADG100 and LMP in each week derived from $\hat{\mathbf{G}}$. Therefore, the cumulated breeding value for RFI during the entire test can be obtained as $\sum_{w=1}^{10} \hat{a}_{i w}^{\mathrm{RFI}}$. Hence, RFI was derived directly from the covariance function that resulted from the Horizontal multitrait random regression model analysis. This leads to a consistent measure of feed efficiency, as the covariance functions are based on a set of consistent parameters, which are estimated simultaneously (Jensen, 2013; Strathe et al., 2014). Standard errors of population parameters were estimated following Fischer et al. (2004) and were in the range of 0.01 to 0.02 for heritabilities and 0.01 to 0.12 for genetic or phenotypic correlations. Standard errors were mostly in the lower range due to the large number of animals and records used in the study. Therefore, SE are not presented to ease the presentation of the results and increase readability of the manuscript.

\section{RESULTS}

Table 1 shows the means, CV, minimum, and maximum values for FI at different weeks on test and production traits for Danish Duroc, Landrace, and Yorkshire breeds. The results illustrate that FI increases with weeks on test with FI in wk 1 being 1,358, 1,350, or 1,336 g/d compared with $3,473,3,243$, or $3,023 \mathrm{~g} / \mathrm{d}$ at the last week on test for Duroc, Landrace, and Yorkshire, respectively. Furthermore, Duroc showed numerically larger FI compared with Landrace and Yorkshire, which might be related to the 8 and $12 \%$ greater ADG100, 4 and $9 \%$ greater ADG30, or 2 and $1 \%$ less LMP, respectively. 
Table 2. Genetic parameters for longitudinal traits of feed intake and residual feed intake in Danish Duroc, Landrace, and Yorkshire breeds

\begin{tabular}{|c|c|c|c|c|c|c|c|c|c|c|c|c|c|c|c|}
\hline \multirow[b]{3}{*}{ Week $^{1}$} & \multicolumn{15}{|c|}{ Breed $^{2}$} \\
\hline & \multicolumn{5}{|c|}{ Duroc } & \multicolumn{5}{|c|}{ Landrace } & \multicolumn{5}{|c|}{ Yorkshire } \\
\hline & $h^{2^{n^{n}}}$ & $\sigma_{a}^{2^{m}}$ & $h^{2^{2 n h}}$ & $\sigma_{a}^{2^{R+r}}$ & Prop., \% & $h^{2^{\mathrm{F}^{\mathrm{H}}}}$ & $\sigma_{a}^{2^{n}}$ & $h^{2^{2 n}}$ & $\sigma_{a}^{2^{k n}}$ & Prop., \% & $h^{2^{n^{n}}}$ & $\sigma_{a}^{2^{\mathrm{n}}}$ & $h^{2^{R H}}$ & $\sigma_{a}^{2^{k n}}$ & Prop., \% \\
\hline 1 & 0.13 & 14,229 & 0.09 & 10,725 & 75 & 0.13 & 18,914 & 0.09 & 14,275 & 75 & 0.22 & 10,847 & 0.20 & 9,842 & 91 \\
\hline 2 & 0.15 & 16,550 & 0.08 & 9,639 & 58 & 0.14 & 17,731 & 0.08 & 10,697 & 60 & 0.21 & 10,895 & 0.16 & 8,198 & 75 \\
\hline 3 & 0.18 & 19,482 & 0.07 & 9,049 & 46 & 0.17 & 19,036 & 0.08 & 9,303 & 49 & 0.22 & 12,641 & 0.12 & 7,375 & 58 \\
\hline 4 & 0.18 & 21,691 & 0.05 & 7,793 & 36 & 0.18 & 21,422 & 0.07 & 8,558 & 40 & 0.22 & 15,205 & 0.09 & 6,458 & 42 \\
\hline 5 & 0.18 & 23,018 & 0.04 & 6,012 & 26 & 0.20 & 24,228 & 0.06 & 7,809 & 32 & 0.22 & 18,263 & 0.06 & 5,182 & 28 \\
\hline 6 & 0.18 & 24,556 & 0.03 & 5,017 & 20 & 0.21 & 27,337 & 0.05 & 6,943 & 25 & 0.22 & 21,986 & 0.03 & 3,760 & 17 \\
\hline 7 & 0.18 & 28,511 & 0.04 & 7,142 & 25 & 0.21 & 31,133 & 0.04 & 6,384 & 21 & 0.21 & 27,018 & 0.02 & 2,887 & 11 \\
\hline 8 & 0.19 & 36,796 & 0.06 & 14,343 & 39 & 0.22 & 36,142 & 0.04 & 6,933 & 19 & 0.22 & 34,222 & 0.02 & 3,648 & 11 \\
\hline 9 & 0.19 & 48,580 & 0.09 & 25,644 & 53 & 0.21 & 42,471 & 0.04 & 9,095 & 21 & 0.23 & 43,997 & 0.03 & 6,943 & 16 \\
\hline 10 & 0.18 & 61,142 & 0.10 & 38,086 & 62 & 0.17 & 49,559 & 0.04 & 12,642 & 26 & 0.23 & 55,539 & 0.05 & 12,643 & 23 \\
\hline P1 & 0.45 & 429,067 & 0.13 & 188,132 & 44 & 0.45 & 462,888 & 0.19 & 223,367 & 48 & 0.50 & 297,753 & 0.27 & 167,801 & 56 \\
\hline $\mathrm{P} 2$ & 0.47 & 882,904 & 0.13 & 339,630 & 38 & 0.51 & 885,721 & 0.09 & 172,210 & 19 & 0.54 & 842,927 & 0.05 & 95,044 & 11 \\
\hline TP & 0.55 & $1,929,458$ & 0.07 & 446,532 & 23 & 0.60 & $2,367,053$ & 0.14 & 614,235 & 26 & 0.62 & $1,811,213$ & 0.09 & 313,367 & 17 \\
\hline
\end{tabular}

${ }^{1} \mathrm{P} 1=$ period from wk 1 to $5 ; \mathrm{P} 2=$ period from wk 6 to $10 ; \mathrm{TP}=$ period from wk 1 to 10.

$2 h^{2^{\mathrm{F}}}=$ heritability for feed intake; $h^{2^{\mathrm{RH}}}=$ heritability for residual feed intake; $\sigma_{a}^{2^{\mathrm{H}}}=$ genetic variance for feed intake; $\sigma_{a}^{2^{\mathrm{kn}}}=$ genetic variance for residual feed intake; Prop. $=$ proportion of $\sigma_{a}^{2^{\mathrm{kn}}}$ to $\sigma_{a}^{2^{\mathrm{H}}}$.

\section{Genetic Background of Feed Intake and Feed Efficiency during Growth}

Estimates of genetic variances and heritabilities of weekly and cumulative FI and RFI traits are presented in Table 2 for Duroc, Landrace, and Yorkshire. In Duroc, FI heritability increased from 0.13 to 0.18 from the first to the third week on test. However, from the first to the last week on test, the genetic variance increased more than 4-fold. This increase was more substantial from wk 7 onwards. For genetic RFI, the heritability and genetic variance showed a quadratic development during the test period, with genetic variance reduced from 10,725 in wk 1 to 5,017 in wk 6 and increased to 38,086 by end of the test period. This resulted in the period from wk 6 to $10(\mathbf{P 2})$ having about twice as large a genetic variance for FI and RFI compared with the period from wk 1 to 5 (P1), with no significant differences in heritabilities. In Landrace, the heritability of FI increased from the first $(0.13)$ to the eighth week $(0.22)$ and then decreased to 0.17 by end of the test period. This was despite the constant increase in genetic variance from the second $(17,731)$ to the last week $(49,559)$ on test. For RFI, the heritability estimate decreased from 0.09 at the first week to 0.04 by wk 7 and it remained constant until the end of the test period. However, the genetic variance had a quadratic development, with reduction from the first $(14,275)$ to the seventh $(6,384)$ week on test followed by an increase towards the end of test $(12,642)$. Feed intake in $\mathrm{P} 2$ compared with $\mathrm{P} 1$ had greater heritability ( 0.51 vs. 0.45 , respectively) and genetic variance $(885,721$ vs. 462,888 , respectively[). On the other hand, RFI had moderate heritability in P1 and low heritability in P2. In Yorkshire, heritabilities of FI were stable with slight fluctuation around a heritability of 0.22 . However, the genetic variance increased during the test, resulting in an increase from 10,847 to 55,539 . In addition, RFI heritability and genetic variance showed quadratic development across the test period, with reduction from the first to the seventh week on test and increasing thereafter. This resulted in RFI having a larger heritability and genetic variance in P1 compared with P2, which was in contrast to FI. The proportion of genetic variance in FI explained by RFI showed a quadratic development, with largest proportion (75 to 91\%) in the beginning of the test and lowest in wk 6 in Duroc or wk 8 in the maternal line breeds followed by increase again towards the end of the test period.

\section{Correlations among Feed Intake or Feed Efficiency Traits during Test Period}

The genetic and phenotypic correlations among FI or RFI records during the test period are presented in Supplementary Tables S1-S6 (see the online version of the article at http://journalofanimalscience.org) for the Duroc, Landrace, and Yorkshire breeds. Figure 1 illustrates the genetic correlations detailed in the Supplementary Tables S1, S2, S3. Figure 1 shows the genetic correlation of FI at wk 5 and 10 with other weeks on test for the different breeds in the study. Week five had strong positive genetic correlations with the other weeks on test and decreased with the increase in the distance between weeks. Week ten showed positive low to moderate 


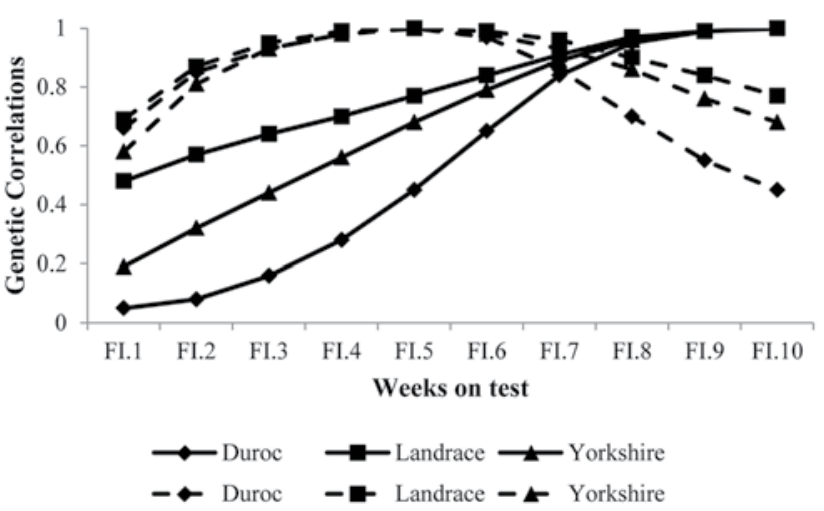

Figure 1. Genetic correlation of feed intake (FI) at wk 10 (solid lines) and at wk 5 (dotted lines) with FI records of different weeks on test (FI. $\{$ week $\}$ ) in Duroc, Landrace, and Yorkshire pigs.

genetic correlations with the early weeks on test in Duroc, and these correlations were positive moderate to large in the dam line breeds. The genetic correlations between 2 subsequent FI records were positive and large ( 0.93 to 0.99 ) in all breeds. However, the correlations dropped as the distance between weeks on test increased, resulting in the lowest correlations between FI in wk 1 with last week on test in the Duroc (0.05), Landrace (0.48), and Yorkshire (0.19) breeds. Phenotypic correlations were in the same direction but with lower magnitude than the genetic correlations. In all breeds, FI.TP had strong genetic correlations with FI in individual weeks on test as well as with FI.P1 and FI.P2. The genetic correlations of FI.TP with FI at individual weeks on test reached its maximum by wk 6 or 7 on test in all breeds in the study. The FI.P1 had systematically stronger correlations with FI in the early weeks (wk 1 to 5) on test whereas FI.P2 had stronger correlations with FI in the late weeks (wk 6 to 10) on test. The FI.TP had stronger genetic correlations with FI.P2 compared with FI.P1 in all breeds.

Genetic RFI at adjacent weeks on test had large genetic correlations ( 0.83 to 1.00$)$ with each other in all breeds. The genetic correlations between RFI measured in different weeks decreased by the increase in the distance between weeks. The genetic correlations among genetic RFI in the early (wk 1 to 5) weeks on test were positive and large as well as among the late (wk 6 to 10) weeks on test. Furthermore, the Duroc breed had low to moderate negative genetic correlations between RFI estimates in the early and late weeks on test, which were substantially different from Landrace with low to moderately positive correlations. For Yorkshire, only the genetic correlations of RFI in wk 9 and 10 had low to moderately negative correlations with RFI in the early weeks on test. In addition, in Duroc RFI.TP had a positive but moderate genetic correlation with RFI in the early weeks on test followed by positive and strong correlations with the other weeks. In Landrace, RFI.TP had large positive genetic correlations with all weeks on test. For Yorkshire, the genetic correlations of RFI. $\mathrm{TP}$ with different weeks on test were positive and large except for low to moderate positive correlations with wk 9 or 10 . The genetic correlations of RFI.TP with individual weeks on test reached its maximum by wk 6 or 7 on test in all breeds. In addition, the RFI.TP had larger genetic correlations with RFI.P2 than with RFI. $\mathrm{P} 1$ in all 3 breeds. Furthermore, the maximum genetic correlation of RFI.P1 was with wk 4 and for RFI.P2 it was with wk 7 or 8 on test in all breeds.

The genetic correlations between intercept and quadratic effects were different between Duroc $(-0.04)$ and the dam line breeds of Landrace (0.12) and Yorkshire (0.18). Furthermore, correlations between linear and quadratic effects was different in Landrace (0.19) compared with the other 2 breeds of Duroc (0.49) and Yorkshire (0.41; Supplementary Table S7; see the online version of the article at http://journalofanimalscience.org).

\section{Characteristics of Single Recorded Production Traits}

The heritability and genetic variances along with genetic and phenotypic correlations for production traits for the 3 breeds are presented in Table 3 . Heritability of ADG100 was moderate in all 3 breeds, with dam lines having larger estimates than Duroc. Heritability of LMP was moderate in Duroc but large in the dam line breeds. Average daily gain from birth to $30 \mathrm{~kg} \mathrm{BW}$ had moderate heritability in all 3 breeds.

The genetic correlation between ADG100 and LMP was negative but low ( -0.13$)$ for Duroc and moderately negative in Landrace $(-0.31)$ and Yorkshire $(-0.32)$. Average daily gain from 30 to $100 \mathrm{~kg} \mathrm{BW}$ and ADG30 showed moderate positive genetic correlations in all 3 breeds. The genetic correlations between LMP and ADG30 were slightly negative in all 3 breeds, with stronger negative correlations in the dam line breeds.

\section{Correlations among Feed Intake or Residual Feed Intake with Single Recorded Traits}

Genetic and phenotypic correlations of FI and genetic RFI with production traits are presented in Tables 4 and 5 for all breeds. The genetic correlations between FI and ADG100 were positive and increased from the first to seventh week on test from 0.48 to 0.85 in Duroc. The change was similar in Landrace, but in Yorkshire, the increase was from 0.23 to 0.92 . However, it decreased to 0.58 in the last week on test in Duroc whereas it plateaued in the dam line breeds. Phenotypic correlations between FI and ADG100 ranged from positive low to moderate, with the first week on test as the lowest correlation in all 3 breeds. In Duroc, LMP and FI started with positive low genetic correlation, and thereafter, correlations decreased 
Table 3. Heritability $\left(h^{2}\right)$ and genetic variances $\left(\sigma_{a}^{2}\right)$ along with genetic (below diagonal) and phenotypic (above diagonal) correlations for production traits of ADG from birth to $30 \mathrm{~kg} \mathrm{BW}$ (ADG30) and from 30 to $100 \mathrm{~kg} \mathrm{BW}$ (ADG100) as well as lean meat percentage (LMP) in Danish Duroc, Landrace, and Yorkshire breeds

\begin{tabular}{|c|c|c|c|c|c|c|c|c|c|c|c|c|c|c|c|}
\hline \multirow[b]{4}{*}{ Traits } & \multicolumn{15}{|c|}{ Breed } \\
\hline & \multicolumn{5}{|c|}{ Duroc } & \multicolumn{5}{|c|}{ Landrace } & \multicolumn{5}{|c|}{ Yorkshire } \\
\hline & \multirow[b]{2}{*}{$h^{2}$} & \multirow[b]{2}{*}{$\sigma_{a}^{2}$} & \multicolumn{3}{|c|}{ Correlations } & \multirow[b]{2}{*}{$h^{2}$} & \multirow[b]{2}{*}{$\sigma_{a}^{2}$} & \multicolumn{3}{|c|}{ Correlations } & \multirow[b]{2}{*}{$h^{2}$} & \multirow[b]{2}{*}{$\sigma_{a}^{2}$} & \multicolumn{3}{|c|}{ Correlations } \\
\hline & & & ADG100 & LMP & ADG30 & & & ADG100 & LMP & ADG30 & & & ADG100 & LMP & ADG30 \\
\hline ADG100 & 0.24 & 2,008 & & -0.03 & 0.09 & 0.34 & 2,857 & & -0.15 & 0.12 & 0.34 & 2,904 & & -0.15 & 0.14 \\
\hline LMP & 0.37 & 0.25 & $7-0.13$ & & 0.02 & 0.62 & 0.76 & $9-0.31$ & & -0.04 & 0.60 & 0.27 & $9-0.32$ & & -0.06 \\
\hline ADG30 & 0.22 & 254.9 & 0.37 & -0.01 & & 0.25 & 238.0 & 0.36 & -0.11 & & 0.22 & 194.6 & 0.42 & -0.16 & \\
\hline
\end{tabular}

towards negative moderate $(-0.26)$. The genetic correlations of LMP and FI in dam line breeds were negative and of larger magnitude than in Duroc. In Landrace, genetic correlations of LMP and FI changed from being weakly negative at the first week on test to large negative correlations at later weeks on test. In Yorkshire, the genetic correlations were moderate negative in the early stages and strong negative at the later stages of the test period. The genetic correlation of the cumulated FI traits (the period from wk 1 to $10, \mathrm{P} 1$, and $\mathrm{P} 2$ ) with the ADG100 trait was positive and large, and FI.P2 had greater correlation than FI.P1 in all breeds. Genetic correlation of LMP with FI.P1 was negative and low $(-0.06)$ in contrast to being negative and moderate with FI.P2 $(-0.26)$ in Duroc. Furthermore, dam line breeds showed negative and low genetic correlations with FI.P1 in addition to negative and strong genetic correlations with FI.P2. The ADG30 showed stronger genetic correlations with FI.P1 ( 0.34 to 0.51$)$ than with FI.P2 (0.13 to 0.42$)$ in all breeds. Phenotypic correlations of LMP and FI were negative low and close to 0 in Duroc, with the dam line breeds showing negative low to moderate phenotypic correlations. Average daily gain from birth to $30 \mathrm{~kg} \mathrm{BW}$ and FI showed varying genetic correlations during the test period. In Duroc, the correlations were moderately positive, ranging from 0.22 to 0.38 until wk 7 and thereafter reduced towards zero correlation with the last week on test. In Landrace, the correlations were moderate, ranging from 0.19 to 0.39 . In Yorkshire, correlations were stronger than the other breeds, with positive moderate correlations in the early and late weeks and positive strong in the rest of the weeks on test.

Genetic correlations of ADG30 and RFI were low positive in the early weeks on test but low to moderate negative by end of the test period for the Duroc and Landrace breeds. For the Yorkshire breed, the genetic correlations of ADG30 and RFI had a quadratic development, with being positive but low in early weeks then positive moderate by middle of the test and becoming slightly negative towards the end of test period. The genetic correlations of ADG30 with RFI.P1 were positive and low (0.12 to 0.32$)$ in all breeds. However,
RFI.P2 had negative and low genetic correlations with ADG30 in Duroc $(-0.23)$ and Landrace $(-0.04)$ compared with positive and low genetic correlations $(0.10)$ in Yorkshire. This was followed by RFI.TP and ADG30 having negative and low $(-0.11)$ genetic correlations in Duroc, positive and low (0.05) genetic correlations in Landrace, and positive and moderate $(0.29)$ genetic correlations in Yorkshire. Phenotypic correlations between RFI and ADG100 were moderately negative with a quadratic development and reached their peak by wk 5 on test in Duroc. In Landrace, the correlations were positive but low at the early weeks on test and moderate afterwards. However, Yorkshire showed negative correlations, with low correlations in the beginning of the test and moderate correlations later in the test. Lean meat percentage and RFI had varying phenotypic correlations between different breeds, with Duroc having negative low correlations in the early weeks and positive low correlations in the late weeks on test. In Landrace, the correlations were negative and increased by weeks on test, resulting in negative low to moderate phenotypic correlations. However, Yorkshire showed constant low positive correlations throughout the test period. Average daily gain from birth to $30 \mathrm{~kg} \mathrm{BW}$ and RFI had very low phenotypic correlations in all breeds.

Genetic regression coefficients for ADG100 and LMP can provide a biological explanation to further reveal the underlying dynamics of RFI over the growth period (Supplementary Table S8; see the online version of the article at http://journalofanimalscience. org). The partial genetic regression coefficients of ADG100 increased and those for LMP decreased by weeks on test. This resulted in ADG100 having a larger and LMP having less partial regression coefficient in P2 than in P1. Duroc showed larger partial regression coefficients for ADG100 but less for LMP in P1 and $\mathrm{P} 2$ compared with dam line breeds.

\section{DISCUSSION}

In the present study, the Horizontal model was proposed and shown to be feasible for dissecting the ge- 
Table 4. Genetic $\left(r_{\mathrm{g}}\right)$ and phenotypic $\left(r_{\mathrm{p}}\right)$ correlations of feed intake (FI) during wk 1 to 10 on test (FI. \{week\}) with ADG from birth to $30 \mathrm{~kg} \mathrm{BW}$ (ADG30) and from 30 to $100 \mathrm{~kg} \mathrm{BW}$ (ADG100) as well as lean meat percentage (LMP) in Danish Duroc, Landrace, and Yorkshire breeds

\begin{tabular}{|c|c|c|c|c|c|c|c|c|c|c|c|c|c|c|c|c|c|c|}
\hline \multirow[b]{4}{*}{ Traits ${ }^{1}$} & \multicolumn{18}{|c|}{ Breed } \\
\hline & \multicolumn{6}{|c|}{ Duroc } & \multicolumn{6}{|c|}{ Landrace } & \multicolumn{6}{|c|}{ Yorkshire } \\
\hline & \multicolumn{2}{|c|}{ ADG100 } & \multicolumn{2}{|c|}{ LMP } & \multicolumn{2}{|c|}{ ADG30 } & \multicolumn{2}{|c|}{ ADG100 } & \multicolumn{2}{|c|}{ LMP } & \multicolumn{2}{|c|}{ ADG30 } & \multicolumn{2}{|c|}{ ADG100 } & \multicolumn{2}{|c|}{ LMP } & \multicolumn{2}{|c|}{ ADG30 } \\
\hline & $r_{\mathrm{g}}$ & $r_{\mathrm{p}}$ & $r_{\mathrm{g}}$ & $r_{\mathrm{p}}$ & $r_{\mathrm{g}}$ & $r_{\mathrm{p}}$ & $r_{\mathrm{g}}$ & $r_{\mathrm{p}}$ & $r_{\mathrm{g}}$ & $r_{\mathrm{p}}$ & $r_{\mathrm{g}}$ & $r_{\mathrm{p}}$ & $r_{\mathrm{g}}$ & $r_{\mathrm{p}}$ & $r_{\mathrm{g}}$ & $r_{\mathrm{p}}$ & $r_{\mathrm{g}}$ & $r_{\mathrm{p}}$ \\
\hline FI.1 & 0.48 & 0.09 & 0.05 & 0.00 & 0.28 & 0.06 & 0.49 & 0.11 & -0.11 & -0.04 & 0.19 & 0.04 & 0.23 & 0.07 & -0.27 & -0.11 & 0.23 & 0.05 \\
\hline FI.2 & 0.64 & 0.14 & 0.00 & -0.01 & 0.36 & 0.08 & 0.63 & 0.15 & -0.20 & -0.07 & 0.30 & 0.06 & 0.45 & 0.13 & -0.34 & -0.13 & 0.43 & 0.10 \\
\hline FI. 3 & 0.73 & 0.17 & -0.05 & -0.02 & 0.38 & 0.09 & 0.71 & 0.19 & -0.29 & -0.11 & 0.36 & 0.08 & 0.62 & 0.19 & -0.38 & -0.15 & 0.53 & 0.12 \\
\hline FI.4 & 0.80 & 0.19 & -0.10 & -0.03 & 0.38 & 0.08 & 0.77 & 0.21 & -0.36 & -0.13 & 0.38 & 0.09 & 0.73 & 0.22 & -0.42 & -0.17 & 0.58 & 0.14 \\
\hline FI. 5 & 0.86 & 0.21 & -0.15 & -0.04 & 0.36 & 0.08 & 0.80 & 0.23 & -0.42 & -0.16 & 0.39 & 0.09 & 0.82 & 0.25 & -0.45 & -0.18 & 0.58 & 0.14 \\
\hline FI.6 & 0.89 & 0.21 & -0.20 & -0.05 & 0.31 & 0.06 & 0.83 & 0.25 & -0.48 & -0.19 & 0.37 & 0.10 & 0.89 & 0.27 & -0.47 & -0.18 & 0.56 & 0.14 \\
\hline FI.7 & 0.85 & 0.21 & -0.25 & -0.06 & 0.22 & 0.05 & 0.85 & 0.26 & -0.53 & -0.21 & 0.33 & 0.09 & 0.92 & 0.28 & -0.49 & -0.19 & 0.51 & 0.13 \\
\hline FI. 8 & 0.76 & 0.19 & -0.27 & -0.07 & 0.13 & 0.03 & 0.84 & 0.26 & -0.56 & -0.22 & 0.29 & 0.08 & 0.92 & 0.28 & -0.49 & -0.19 & 0.43 & 0.11 \\
\hline FI.9 & 0.66 & 0.17 & -0.27 & -0.07 & 0.05 & 0.01 & 0.82 & 0.25 & -0.58 & -0.22 & 0.24 & 0.07 & 0.90 & 0.28 & -0.48 & -0.19 & 0.36 & 0.10 \\
\hline FI.10 & 0.58 & 0.14 & -0.26 & -0.06 & 0.00 & 0.00 & 0.79 & 0.22 & -0.58 & -0.20 & 0.19 & 0.05 & 0.86 & 0.26 & -0.46 & -0.18 & 0.29 & 0.08 \\
\hline FI.P1 & 0.75 & 0.28 & -0.06 & -0.03 & 0.37 & 0.13 & 0.72 & 0.31 & -0.29 & -0.18 & 0.34 & 0.12 & 0.63 & 0.29 & -0.40 & -0.24 & 0.51 & 0.18 \\
\hline FI.P2 & 0.77 & 0.30 & -0.26 & -0.10 & 0.13 & 0.04 & 0.84 & 0.40 & -0.56 & -0.33 & 0.28 & 0.12 & 0.92 & 0.44 & -0.49 & -0.30 & 0.42 & 0.17 \\
\hline FI.TP & 0.87 & 0.37 & -0.21 & -0.09 & 0.26 & 0.10 & 0.83 & 0.42 & -0.47 & -0.31 & 0.32 & 0.14 & 0.88 & 0.45 & -0.50 & -0.33 & 0.50 & 0.21 \\
\hline
\end{tabular}

${ }^{1} \mathrm{FI} . \mathrm{P} 1=$ feed intake during wk 1 to $5 ; \mathrm{FI} . \mathrm{P} 2=$ feed intake during wk 6 to $10 ; \mathrm{FI} . \mathrm{TP}=$ feed intake during wk 1 to 10.

netic background of the feed efficiency complex when feed efficiency is studied over the growth period. The model allowed flexible multivariate analysis of longitudinal data with single recorded traits by allowing separate estimation of environmental variance for each longitudinal time point without forcing any structure on the environmental variances and covariances in the model. Such assumptions would fit many situations where longitudinal and single traits are recorded in animal breeding programs. In common longitudinal genetic analysis, the random regression method decomposes the environmental variance into permanent effects over repeated records and temporal effects affecting each record specifically. Hence, the between-animal variance in random regression models includes at least 2 sets of random regression coefficients for each animal, representing additive genetic and permanent environmental effects, respectively. However, this leads to problems in joint analysis where some traits are repeatedly recorded and others are single recorded traits, because of the partitioning of the environmental variance between the permanent and temporal residual variance for the single recorded trait. In the Horizontal approach, the fixed effects that are common to several traits can also be modeled to have a common effect across the longitudinal time points by specifying common effects. Furthermore, the Horizontal model can provide estimates of environmental variance and covariance for each week on test. The Horizontal model has the advantage of easy modeling of the environmental covariances between longitudinal and single recorded traits. This allows more accurate estimation of genetic merits of animals, as mis-specifying environmental covariance structure can lead to biased estimation of genetic variances and covariances, for example, Schnyder et al. (2002).

\section{Genetic Background of Feed Intake}

The genetic influence on FI changed during the growth of the pigs. Increasing genetic variance of FI with advancing week on test was observed for all breeds, with Duroc having larger variances than Landrace and Yorkshire despite heritabilities similar to or lower than the dam line breeds. Increase in mean and SD of FI during the test period along with the change in the genetic parameters illustrate the change in FI capacity of animals during growth. In addition, numerically greater FI in Duroc compared with dam line breeds could be due to greater ADG100 and less LMP than Landrace and Yorkshire. This indicates a larger feed intake capacity in the Duroc breed. In Danish pig breeding, Duroc is a terminal sire line with strong selection pressure for feed efficiency, growth, and lean meat production; however, Landrace and Yorkshire are dam line breeds with the primary selection objective of improvement in maternal traits but still a substantial emphasis on feed efficiency and growth. Figure 1 shows smooth results also towards the borders of the data, suggesting no border effects, which are sometimes seen in random regression models using Legendre polynomials, for example, David et al. (2015). Cai et al. (2011) reported heritabilities of 0.10 to 0.37 for daily FI during the test period using random regression with second-order Legendre polynomials of age in boars of Yorkshire pigs. In addition, 
Table 5. Genetic $\left(r_{\mathrm{g}}\right)$ and phenotypic $\left(r_{\mathrm{p}}\right)$ correlations of residual feed intake (RFI) during wk 1 to 10 on test (RFI. \{week\}) with ADG from birth to $30 \mathrm{~kg} \mathrm{BW}$ (ADG30) and from 30 to $100 \mathrm{~kg} \mathrm{BW} \mathrm{(ADG100)} \mathrm{as} \mathrm{well} \mathrm{as} \mathrm{lean}$ meat percentage (LMP) in Danish Duroc, Landrace, and Yorkshire breeds

\begin{tabular}{|c|c|c|c|c|c|c|c|c|c|c|c|c|c|c|c|c|c|c|}
\hline \multirow[b]{4}{*}{ Traits $^{1}$} & \multicolumn{18}{|c|}{ Breed } \\
\hline & \multicolumn{6}{|c|}{ Duroc } & \multicolumn{6}{|c|}{ Landrace } & \multicolumn{6}{|c|}{ Yorkshire } \\
\hline & \multicolumn{2}{|c|}{ ADG100 } & \multicolumn{2}{|c|}{ LMP } & \multicolumn{2}{|c|}{ ADG30 } & \multicolumn{2}{|c|}{ ADG100 } & \multicolumn{2}{|c|}{ LMP } & \multicolumn{2}{|c|}{ ADG30 } & \multicolumn{2}{|c|}{ ADG100 } & \multicolumn{2}{|c|}{ LMP } & \multicolumn{2}{|c|}{ ADG30 } \\
\hline & $r_{\mathrm{g}}$ & $r_{\mathrm{p}}$ & $r_{\mathrm{g}}$ & $r_{\mathrm{p}}$ & $r_{\mathrm{g}}$ & $r_{\mathrm{p}}$ & $r_{\mathrm{g}}$ & $r_{\mathrm{p}}$ & $r_{\mathrm{g}}$ & $r_{\mathrm{p}}$ & $r_{\mathrm{g}}$ & $r_{\mathrm{p}}$ & $r_{\mathrm{g}}$ & $r_{\mathrm{p}}$ & $r_{\mathrm{g}}$ & $r_{\mathrm{p}}$ & $r_{\mathrm{g}}$ & $r_{\mathrm{p}}$ \\
\hline RFI.1 & 0.00 & -0.26 & 0.00 & -0.05 & 0.12 & 0.02 & 0.00 & 0.11 & 0.00 & -0.04 & 0.01 & 0.04 & 0.00 & -0.08 & 0.00 & 0.04 & 0.14 & 0.03 \\
\hline RFI.2 & 0.00 & -0.36 & 0.00 & -0.04 & 0.16 & 0.03 & 0.00 & 0.15 & 0.00 & -0.07 & 0.09 & 0.06 & 0.00 & -0.18 & 0.00 & 0.04 & 0.27 & 0.05 \\
\hline RFI.3 & 0.00 & -0.42 & 0.00 & -0.03 & 0.17 & 0.02 & 0.00 & 0.19 & 0.00 & -0.11 & 0.15 & 0.08 & 0.00 & -0.27 & 0.00 & 0.04 & 0.35 & 0.06 \\
\hline RFI.4 & 0.00 & -0.46 & 0.00 & -0.01 & 0.15 & 0.02 & 0.00 & 0.21 & 0.00 & -0.13 & 0.17 & 0.09 & 0.00 & -0.33 & 0.00 & 0.04 & 0.41 & 0.06 \\
\hline RFI.5 & 0.00 & -0.48 & 0.00 & 0.01 & 0.09 & 0.01 & 0.00 & 0.23 & 0.00 & -0.16 & 0.17 & 0.09 & 0.00 & -0.37 & 0.00 & 0.04 & 0.44 & 0.05 \\
\hline RFI.6 & 0.00 & -0.48 & 0.00 & 0.03 & -0.03 & 0.00 & 0.00 & 0.25 & 0.00 & -0.19 & 0.14 & 0.10 & 0.00 & -0.39 & 0.00 & 0.04 & 0.44 & 0.04 \\
\hline RFI.7 & 0.00 & -0.47 & 0.00 & 0.05 & -0.17 & -0.02 & 0.00 & 0.26 & 0.00 & -0.21 & 0.06 & 0.09 & 0.00 & -0.40 & 0.00 & 0.04 & 0.35 & 0.02 \\
\hline RFI.8 & 0.00 & -0.42 & 0.00 & 0.07 & -0.24 & -0.03 & 0.00 & 0.26 & 0.00 & -0.22 & -0.04 & 0.08 & 0.00 & -0.41 & 0.00 & 0.04 & 0.13 & 0.01 \\
\hline RFI.9 & 0.00 & -0.37 & 0.00 & 0.08 & -0.25 & -0.04 & 0.00 & 0.25 & 0.00 & -0.22 & -0.12 & 0.07 & 0.00 & -0.41 & 0.00 & 0.04 & -0.06 & 0.00 \\
\hline RFI.10 & 0.00 & -0.32 & 0.00 & 0.08 & -0.26 & -0.04 & 0.00 & 0.22 & 0.00 & -0.20 & -0.18 & 0.05 & 0.00 & -0.39 & 0.00 & 0.04 & -0.16 & -0.02 \\
\hline RFI.P1 & 0.00 & -0.61 & 0.00 & -0.04 & 0.15 & 0.03 & 0.00 & 0.31 & 0.00 & -0.18 & 0.12 & 0.12 & 0.00 & -0.41 & 0.00 & 0.06 & 0.32 & 0.08 \\
\hline RFI.P2 & 0.00 & -0.61 & 0.00 & 0.09 & -0.23 & -0.04 & 0.00 & 0.40 & 0.00 & -0.33 & -0.04 & 0.12 & 0.00 & -0.61 & 0.00 & 0.06 & 0.10 & 0.01 \\
\hline RFI.TP & 0.00 & -0.71 & 0.00 & 0.04 & -0.11 & -0.01 & 0.00 & 0.42 & 0.00 & -0.31 & 0.05 & 0.14 & 0.00 & -0.62 & 0.00 & 0.07 & 0.29 & 0.04 \\
\hline
\end{tabular}

${ }^{1}$ RFI.P1 = residual feed intake during wk 1 to 5 ; RFI.P2 = residual feed intake during wk 6 to 10; RFI.TP = residual feed intake during wk 1 to 10.

Schulze et al. (2002) showed an increase in heritabilities of weekly FI from 0.12 to 0.32 from wk 1 to 9 of test for 2 dam lines of PIC Germany (Genus-PIC, Schleswig, Germany). Furthermore, Schnyder et al. (2001) modeled FI of French Landrace and Large White growing pigs with random regression analysis using quadratic polynomials and reported a heritability of 0.09 to 0.25 for this trait during the test period. The above results are in agreement with the heritability found in the current study. Wetten et al. (2012) reported that the heritabilities of daily FI decrease by increasing days on test, ranging from 0.15 to 0.05 in Norwegian Duroc and 0.10 to 0.05 in Norwegian Landrace when using random regression analysis with Legendre polynomials. Do et al. (2013) reported heritabilities of 0.41 for Duroc and 0.48 for Landrace and 0.56 for Yorkshire breeds of the Danish population when analyzing ADFI over the entire test period. This is 26,20 , and $10 \%$ lower than heritabilities for FI.TP reported in the current study for Duroc, Landrace, and Yorkshire, respectively. In addition, lower heritabilities are expected for weekly FI compared with analysis where the traits were measured as ADFI during the entire test period. This is due to larger genetic variance in cumulated or averaged traits over a greater period of time compared with average traits over a short time period, which could be the result of averaging over positively correlated periods. In addition, the averaging reduces the environmental variance. The results indicate the varying genetic background of FI during the growing-finishing period in diverse Danish pig breeds.

Genetic and phenotypic correlations between FI records were reduced as the time interval between mea- surements increased. This pattern was also observed by Schulze et al. (2002) for FI of nucleus boars during the growing-finishing period. Genetic correlations of FI at wk 5 and FI at wk 10 with other weeks on test illustrate that FI has a dynamic genetic background in each breed. However, it should be considered that Legendre polynomials of age used for Duroc were not equidistance with those of the dam line breeds in the later stages. This could be due to larger BW growth in Duroc, which results in finishing the test at younger ages compared with the dam lines. Significant deviation from unity was observed for genetic correlations of FI.TP with different weeks on test, indicating that selection for cumulated FI does not use the dynamics of genetic effects on this trait during the growth period. Lower genetic correlations among FI records in Duroc compared with Landrace may reflect breed differences in the development of FI capacity over the test period. Schnyder et al. (2001) reported that the genetic covariances between intercept, linear, and quadratic regression coefficients of FI were different between French Landrace and Large White breeds. This is in agreement with the results of the current study. These results suggest that the genetic influence on FI capacity varies among the breeds included in this study.

\section{Genetic Background of Production Traits}

Heritability for ADG30 in Duroc (0.22), Landrace $(0.25)$, and Yorkshire (0.22) were larger than the heritability of 0.05 for BW at weaning in terminal sire Duroc pigs reported by Jiao et al (2014). Heritability of ADG100 in Duroc (0.24), Landrace (0.34), and Yorkshire (0.34) were 
lower than those reported by Do et al. (2013) for Danish Duroc (0.32), Landrace (0.54), and Yorkshire (0.47). This could be due to analyzing only the test station data set of the Danish pigs in the study of Do et al. (2013) compared with the current study, which includes data from the nucleus breeder herds as well. The heritability of LMP in Duroc (0.37) was substantially lower than heritability for ultrasound measured back fat for Danish Duroc (0.54) reported by Do et al. (2013). However, in Landrace (0.62) and Yorkshire (0.60), the obtained heritabilities were in agreement with reported heritability found by Do et al. (2013) for ultrasonically measured back fat for Danish Landrace (0.67) and Yorkshire (0.63). Furthermore, Saintilan et al. (2013) reported heritability of ADG and ultrasound lean meat content in Landrace ( 0.26 and 0.66 , respectively), Large White ( 0.33 and 0.60 , respectively), and Pietrain (0.48 and 0.49 , respectively) pigs. In the current study, lower heritabilities of ADG100 and LMP for Duroc were due to lower genetic variance compared with Landrace (30 and 67\%, respectively) and Yorkshire (31 and $8 \%$, respectively). This could be due to lower selection pressure on these traits and subsequently larger fat deposition in Landrace, as it is a maternal line breed. Genetic background of performance traits of BW growth at the weaning and growing-finishing periods as well as LMP was profound for each breed, suggesting the potential for further genetic improvement of these traits. In addition, breed differences in BW growth and LMP were observed between Duroc and maternal line breeds.

Feed intake in individual weeks had positive genetic correlations with ADG100, and these correlations increased by weeks on test. This was followed by an increase in unfavorable genetic correlations between weekly FI and LMP. The results indicate that as pigs grow, more nutrients are allocated for fat deposition. Shirali et al. (2012) showed that late stages of growth (90 to $120 \mathrm{~kg} \mathrm{BW}$ ) are associated with greater nutrient intake and more lipid to protein deposition compared with earlier stages (60 to $90 \mathrm{~kg} \mathrm{BW}$ ) in crossbred growingfinishing pigs. Schulze et al. (2002) observed an increase in the genetic correlation between FI and ADG ( 0.12 to $0.54)$ and back fat ( 0.22 to 0.41$)$ by increasing weeks on test from wk 1 to 9. Furthermore, Schnyder et al. (2002) observed that FI at different weeks on test had positive and large genetic correlations with ADG and moderately negative correlations with carcass lean content in a Large White breed. The above findings are in agreement with the results in the current study. In addition, Schulze et al. (2002) reported moderate favorable correlations of intercept $(0.21)$ and slope $(0.42)$ of FI with ADG and moderate unfavorable correlations of intercept $(0.21)$ and slope (0.31) with back fat thickness. Furthermore, Schnyder et al. (2002) estimated the genetic correlation of intercept, linear, and quadratic regression coefficients for FI with single record performance traits of $\operatorname{ADG}(0.82,0.38$, and 0.63 , respectively) and carcass lean content $(-0.33,-0.55$, and 0.13 , respectively). This indicates that selection for quadratic effects on FI improves not only the BW gain but also lean meat production in pigs through reduction in FI with increasing weeks on test. Strathe et al. (2014) estimated parameters among FI and BW (both recorded longitudinally) for Danish Duroc pigs using a bivariate random regression model with second-order Legendre polynomials. The genetic correlation between FI and growth as average over the entire test period was 0.87 . In the current study, the genetic correlation of cumulated FI during the entire test was also substantially large for all breeds, ranging from 0.83 to 0.88 . Furthermore, moderate favorable genetic correlations between single recorded performance traits of ADG30 and ADG100 suggest that pigs with improved BW growth at weaning time would have superior ADG during the growing-finishing period with no or small side effects on lean meat production. The results suggest that the later stages of growth are associated with higher FI and therefore fat deposition. However, selection for curves of FI can allow improvement in BW growth and LMP followed by reduction in FI at later stages of growth.

\section{Dynamic Genetic Background of Feed Efficiency}

The current study shows that the genetic effects on partial feed efficiency changes over the period of performance testing animals for growth and feed efficiency - and that these dynamics differ between breeds. Heritability of RFI was larger at the beginning and the end of the test period, with wk 6 to 8 being the nadir. Strathe et al. (2014) observed a low heritability estimate range of 0.01 to 0.07 in Duroc breed using a bivariate random regression analysis of cumulative FI and longitudinal BW records during the growth of pigs. Shirali et al. (2014) reported large RFI heritabilities (0.47 to 0.50$)$ at different stages of growth for crossbred pigs grown from 60 to $140 \mathrm{~kg} \mathrm{BW}$. This was substantially larger than the 0.05 to 0.25 heritabilities obtained in the current study for RFI in P1 and P2 in Danish terminal and dam line breeds. This difference could be due to using purebred nucleus animals in this study compared with the crossbred commercial pigs in the study of Shirali et al. (2014). When RFI was calculated for the entire test period, the range of heritabilities was from 0.10 to 0.40 (Cai et al., 2008; Do et al., 2013; Saintilan et al., 2013). The heritabilities of RFI.TP were in the lower range of the above reported heritabilities. In general, RFI is a partial measure of feed efficiency, with the components of feed efficiency related to production and maintenance being excluded; therefore, additive genetic variance in partial feed efficiency is a consequence of the genetic 
correlations between underlying traits that define this trait. Hence, a large proportion of FI explained by RFI in early weeks on test can be due to low genetic correlations of FI with ADG100 and LMP. Furthermore, the largest genetic correlation of FI with ADG100 and LMP were found in wk 4 to 8 in Duroc and wk 4 to 10 in the dam line breeds, resulting in less variation available in FI to be explained by RFI. Shirali et al. (2014) reported an increase in phenotypic variance in residual energy intake as a measure of feed efficiency when energy intake was corrected for lean and fat deposition using phenotypic regression. This was followed by an increase in the proportion of variance in energy intake explained by residual energy intake ( 26 to $32 \%$ ). This could be due to growing animals until an average BW of $140 \mathrm{~kg}$ in above study compared with a BW of 100 $\mathrm{kg}$ in the current study. Larger partial genetic regression coefficient for LMP in P1 compared with P2 indicates that this period is more associated with lean meat deposition, and substantially larger coefficients for ADG100 in $\mathrm{P} 2$ could be due to larger fat deposition in this period. Furthermore, Shirali et al. (2012) showed that early stages of growth (60 to $90 \mathrm{~kg} \mathrm{BW}$ ) are characterized by a lower lipid-to-protein ratio and also a lower $\mathrm{CV}$ in this trait compared with later stages of growth (90 to 120 $\mathrm{kg} \mathrm{BW}$ ), indicating that later stages are associated with fat growth compared with early stages for lean growth deposition. In addition, genetic partial regression coefficients for ADG and LMP suggest that the improved feed efficiency in Duroc is due to more efficient production of lean BW compared with dam line breeds. This indicates that underlying biological factors influencing lean meat deposition such as less FI and more efficiency in lean deposition have key roles in improvement of feed efficiency in pigs. Knap (2012) reported that 35 years of genetic improvement of lean growth rate in pigs has resulted in a $31 \%$ reduction in nitrogen excreted per kilogram of protein produced. Furthermore, better feed efficiency in Landrace can be explained by less fat deposition in this breed compared with Yorkshire. Despite favorable genetic correlations of growth rate and LMP with more efficient animals, the partial measure of feed efficiency indicates that further genetic improvement in efficiency of nutrient utilization can be obtained through selection for genetic RFI. This is due to the fact that genetic RFI is a component of efficiency that is independent of production and maintenance, with contributing factors being digestibility, physical activity, tissue turnover, energy utilization, etc. (Dekkers and Gilbert, 2010). However, selection on phenotypic RFI obtained through conditioning of FI for BW gain and LMP using phenotypic partial regression coefficients would result in disproportional selection pressure on production traits in the breeding program due to the existence of genetic correlations of phenotypic RFI with these production traits as shown by Shirali et al. (2016).

A varying genetic influence on feed efficiency was observed in the current study along with a decrease in the genetic correlations between RFI measured with increasing interval between measurements. This suggests that different genes can be associated with RFI at different stages of growth. Shirali et al. (2013) showed that different QTL influence RFI at different stages of growth in growing-finishing pigs. In addition, Shirali et al. (2014) reported that RFI at 60 to $90 \mathrm{~kg}$ BW was lowly correlated with RFI at 90 to $120 \mathrm{~kg} \mathrm{BW}$ and 120 to $140 \mathrm{~kg} \mathrm{BW}$, supporting the findings of this study.

The definition of RFI used in the current study uses the partial genetic regression coefficient combined with better modeling of the genetic and environmental effects on FI capacity during growth. The partial genetic coefficient ensures that RFI is genetically independent of production traits; however, the phenotypic correlation between these traits exists $\left(\operatorname{cov}\left(y_{\mathrm{RFI}}, y_{p}\right)=\operatorname{cov}\left(y_{\mathrm{FI}}\right.\right.$, $\left.\left.y_{p}\right)-b_{g} \operatorname{var}\left(y_{p}\right)\right)$, which equals $\operatorname{cov}\left(e_{\mathrm{FI}}, w_{p}\right)-\operatorname{cov}\left(g_{\mathrm{FI}}\right.$, $\left.g_{p}\right)\left(1-h_{p}{ }^{2} / h_{p}{ }^{2}\right)$ also shown by Kennedy et al. (1993). The Horizontal model combined with estimation of RFI through covariance functions allows consistent estimation of breeding values for RFI in a population by better modeling the FI capacity of pigs during growth and accurately modeling the genetic and residual variances and covariances between traits. Kennedy et al. (1993) showed that partial phenotypic or genetic regression coefficients can be used for estimating RFI from multitrait analysis. The above study observed that using partial genetic regression would result in lower heritability compared with using partial phenotypic regression. However, the study above suggested that RFI estimated as genetically adjusting FI for production traits would be easier to implement in index selection. When analyzing genetic RFI as in the current study, the heritability for RFI is less related to the environmental correlations between FI and production traits. This is due to the fact that the genetic and phenotypic variances in FI are adjusted by the partial genetic regression coefficients of FI on ADG100 and LMP, which are independent of environmental variances and covariances. Therefore, it results in genetic independence between RFI and production traits, which is favorable for implementation in breeding programs and yields a breeding value that is easy to communicate to practitioners. Selection for improvement in feed efficiency under breeding conditions can be achieved through multiple-trait selection on FI and production traits using appropriate economic weights in a selection index or using RFI and production traits (Kennedy et al., 1993). Furthermore, breeding values for RFI can be defined on a daily basis or integrated over part of or the entire test period (Jensen, 2013; Strathe et al., 2014). In the 
current study, FI was modeled and later conditioned on (correcting for) production traits to yield genetic RFI in each week on test. Hence, it is illustrated that interesting biological knowledge of the dynamics of feed efficiency during growth can be generated from this model. In addition, by influencing the curve of FI and feed efficiency, it is possible to further improve lean meat feed efficiency.

In conclusion, the Horizontal model proposed in this study can be used to analyze repeated records simultaneously together with single recorded traits through specific modeling of the environmental variances and covariances. The Horizontal model allows modeling the change in FI capacity during growth. Clear breed differences between Duroc and dam line breeds in the genetic parameters of the feed efficiency complex were illustrated throughout the period of growth. Feed intake and genetic RFI had varying genetic background during growing-finishing period in pigs. Defining RFI as genetically independent of production traits leads to easily interpretable breeding values.

\section{LITERATURE CITED}

Cai, W., D. S. Casey, and J. C. M. Dekkers. 2008. Selection response and genetic parameters for residual feed intake in Yorkshire swine. J. Anim. Sci. 86:287-298. doi:10.2527/jas.2007-0396

Cai, W., M. S. Kaiser, and J. C. M. Dekkers. 2011. Genetic analysis of longitudinal measurements of performance traits in selection lines for residual feed intake in Yorkshire swine. J. Anim. Sci. 89:1270-1280. doi:10.2527/jas.2010-3107

David, I., J. Ruesche, L. Drouilhet, H. Garreau, and H. Gilbert. 2015. Genetic modeling of feed intake. J. Anim. Sci. 93(3):965-977. doi:10.2527/jas.2014-8507

Dekkers, J. C. M., and H. Gilbert. 2010. Genetic and biological aspect of residual feed intake in pigs. In: Proc. 9th World Congr. Genet. Appl. Livest. Prod., Leipzig, Germany. No. 287.

Do, D. N., A. B. Strathe, J. Jensen, T. Mark, and H. N. Kadarmideen. 2013. Genetic parameters for different measures of feed efficiency and related traits in boars of three pig breeds. J. Anim. Sci. 91:4069-4079. doi:10.2527/jas.2012-6197

Fischer, T. M., A. R. Gilmour, and J. H. van der Werf. 2004. Computing approximate standard errors for genetic parameters derived from random regression models fitted by average information REML. Genet. Sel. Evol. 36(3):363-369. doi:10.1186/12979686-36-3-363

Jensen, J. 2013. Joint estimation for curves for weight, feed intake, rate of gain, and residual feed intake. In: 64th Annu. Meet. Eur. Fed. Anim. Sci., Nantes, France. p. 531.

Jiao, S., C. Maltecca, K. A. Gray, and J. P. Cassady. 2014. Feed intake, average daily gain, feed efficiency, and real-time ultrasound traits in Duroc pigs: I. Genetic parameter estimation and accuracy of genomic prediction. J. Anim. Sci. 92:2377-2386. doi:10.2527/jas.2013-7338

Kennedy, B. W., J. H. van der Werf, and T. H. Meuwissen. 1993. Genetic and statistical properties of residual feed intake. J. Anim. Sci. 71:3239-3250.
Knap, P. W. 2012. Pig breeding for increased sustainability. In: R. A. Meyers, editor, Encyclopedia of sustainability science and technology. Vol. 11. Springer Science+Business Media, LLC, SpringerVerlag New York, Inc., New York, New York. p. 7972-8012.

Littell, R. C., J. Pendergast, and R. Natarajan. 2000. Modelling covariance structure in the analysis of repeated measures data. Stat. Med. 19:1793-1819. doi:10.1002/10970258(20000715)19:13<1793::AID-SIM482>3.0.CO;2-Q

Madsen, P., and J. Jensen. 2014. An user's guide to DMU: A package for analysing multivariate mixed models. Ver. 6, rel. 5.3. Aarhus University, Foulum, Denmark.

Saintilan, R., I. Merour, L. Brossard, T. Tribout, J. Y. Dourmad, P. Sellier, J. Bidanel, J. van Milgen, and H. Gilbert. 2013. Genetics of residual feed intake in growing pigs: Relationships with production traits, and nitrogen and phosphorus excretion traits. J. Anim. Sci. 91:2542-2554. doi:10.2527/jas.2012-5687

Schaeffer, L. R. 2004. Application of random regression models in animal breeding. Livest. Prod. Sci. 86:35-45. doi:10.1016/ S0301-6226(03)00151-9

Schnyder, U., A. Hofer, F. Labroue, and N. Künzi. 2001. Genetic parameters of a random regression model for daily feed intake of performance tested French Landrace and Large White growing pigs. Genet. Sel. Evol. 33:635-658. doi:10.1186/1297-9686-336-635

Schnyder, U., A. Hofer, F. Labroue, and N. Künzi. 2002. Multiple trait model combining random regressions for daily feed intake with single measured performance traits of growing pigs. Genet. Sel. Evol. 34:61-81. doi:10.1186/1297-9686-34-1-61

Schulze, V., R. Roehe, J. L. Bermejo, H. Looft, and E. Kalm. 2002. Genetic associations between observed feed intake measurements during growth, feed intake curve parameters and growing-finishing performances of central tested boars. Livest. Prod. Sci. 73:199-211. doi:10.1016/S0301-6226(01)00254-8

Shirali, M., A. Doeschl-Wilson, C. Duthie, P. W. Knap, E. Kanis, J. A. M. van Arendonk, and R. Roehe. 2014. Estimation of residual energy intake and its genetic background during the growing period in pigs. Livest. Sci. 168:17-25. doi:10.1016/j. livsci.2014.07.018

Shirali, M., A. Doeschl-Wilson, P. W. Knap, C. Duthie, E. Kanis, J. A. M. van Arendonk, and R. Roehe. 2012. Nitrogen excretion at different stages of growth and its association with production traits in growing pigs. J. Anim. Sci. 90:1756-1765. doi:10.2527/ jas.2011-4547

Shirali, M., C. A. Duthie, A. Doeschl-Wilson, P. Knap, E. Kanis, J. A. M. van Arendonk, and R. Roehe. 2013. Novel insight into the genomic architecture of feed and nitrogen efficiency measured by residual energy intake and nitrogen excretion in growing pigs. BMC Genet. 14:121. doi:10.1186/1471-2156-14-121

Shirali, M., P. F. Varley, and J. Jensen. 2016. Bayesian analysis of selection response on linear or ratio feed efficiency traits in pigs. In: Proc. 67th EAAP, Belfast, UK. p. 127.

Strathe, A. B., T. Mark, B. Nielsen, D. N. Do, H. N. Kadarmideen, and J. Jensen. 2014. Deriving genomic breeding values for residual feed intake from covariance functions of random regression models. In: Proc. 10th World Congr. Genet. Appl. Livest. Prod., Vancouver, Canada. Abstr. 116.

Wetten, M., J. Ødegård, O. Vangen, and T. Meuwissen. 2012. Simultaneous estimation of daily weight and feed intake curves for growing pigs by random regression. Animal 6:433-439. doi: $10.1017 / \mathrm{S} 1751731111001832$ 